\title{
Endommagement et écaillage par laser
}

\author{
M. Lacomme, B. Cazalis, J. David, G. Nierat, A. Salères et G. Sibille \\ Commissariat à l'Energie Atomique, Centre d'Etudes de Limeil-Valenton, 94195 Villeneuve Saint Georges \\ cedex, France
}

\begin{abstract}
Résumé - Nous exploitons des resultats expérimentaux sur les effets thermomécaniques induits dans des cibles métalliques après interaction avec le ray omement de la chaîne diagnostic da laser impnlsionnel Phébus. Les simnlations numériques des phénomènes induits par le dépôt d'énergie laser ont été effectuées à l'aide des codes monodimensionnels FCI1 et EFFET1 qui traitent le dépố d'énergie haser, la formation, la propagation et latténuation des contraintes qui en ressultent ainsi que l'endommagement l'écaillage. Cette exploitation a permis de restituer conrectement les contraintes enregistrées par une jauge à quartz et de caler les paramètres de différents modèles de fracture.
\end{abstract}

\begin{abstract}
Phebus laser-driven shock wave is used to study dynamic damage and spallation in aluminium and copper targets. Numerical simulations are performed to calcnlate the ablation pressure, the shock induced and the stress evolution in the targets with the one-dimensional codes FCI1 and EFFET1. We calculate also the spall widths with two damage criteria. Theoretical calculations of the stresses in quartz gauge and the scab thicknesses are in good agreement with the experimental results.
\end{abstract}

\section{Introduction:}

Le laser est une source intense de lumière délivrée en un temps bref. L'irradiation d'une cible par ae rayonnement permet d'atteindre des sollicitations dynamiques à grandes vitesses de déformation, supérieures à $10^{6} \mathrm{~s}^{-1}$.

Nous présentons des résultats expérimentaux d'effets thermomécaniques induits dans des cibles métalliques en alliage d'aluminium AU4G ou en cuivre pur par l'interaction d'un rayonnement laser impulsionnel dans des conditions monodimensionnelles d'écoulement. Ces résultats concernent d'une part des enregistrements de la contrainte sur la face arrière de l'échantillon et d'autre part des mesures se rapportant à l'endommagement des échantillons irradiés.

Les simulations numériques de ces phénomènes ont été effectuées à l'aide des codes monodimensionnels FCI1 et EFFET1.

\section{Description des expériences:}

La chaîne diagnostic du laser Phébus utilisée pour ces expériences délivre une énergie pouvant atteindre 1 kilojoule pour des durées d'irradiation allant de 0,2 à 30 nanosecondes aux longueurs d'onde de 1,05 et 0,53 micromètre. Les surfaces irradiées de 0,5 à quelques $\mathrm{cm}^{2}$ conduisent à des éclairements de $10^{7}$ à quelques $10^{12} \mathrm{~W} / \mathrm{cm}^{2}$.

La contrainte transmise à travers l'échantillon est enregistrée par une jauge à quartz collée sur la face arrière (1) (2).

Par ailleurs, des expériences d'endommagement et d'écaillage sont effectuées dans les mêmes conditions d'éclairement laser sur des cibles nues. Ces échantillons sont ensuite récupérés et analysés par micrographie pour observer les dommages. 
Les matériaux retenus pour ces expériences sont: : - l'alliage d'aluminium AU4G (2017A)

- le cuivre pur OFHC $(\mathrm{Cu}>99,95 \%)$

Le choix des diamètres d'impact par rapport aux épaisseurs des échantillons $(0.3 ; 0.5 ; 1 \mathrm{~mm})$ est tel qu'il permet de conserver une bonne planéité de l'onde dans sa propagation dans le matériau.

La figure 1 présente un exemple d'évolution an cours du temps de la contrainte dans la jauge à quartz accolée à la cible d'aluminium pour les conditions d'irradiation indiquées.

contrainte (kbars)

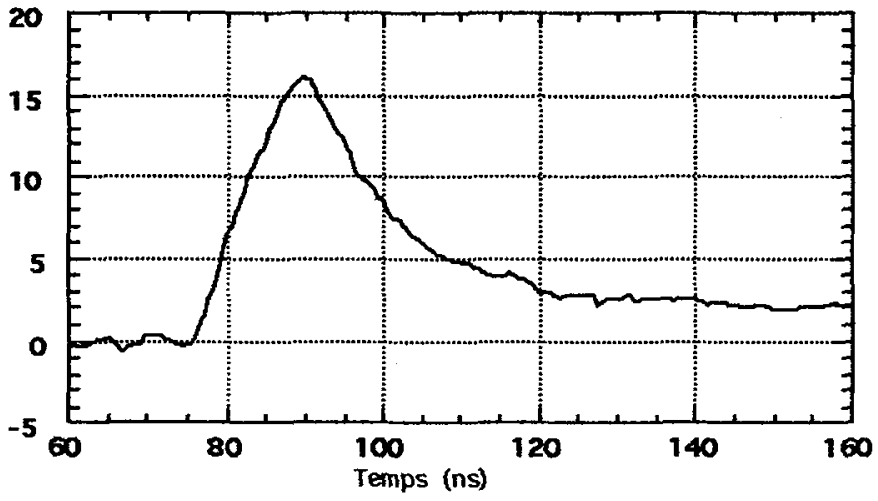

Figure 1:

Evohution temporelle de la contrainte mesuré dans une jauge à quartx collée à l'arrière d'une cible d'alumininm de $0,5 \mathrm{~mm}$ d'épaissenr irradie par une impulsion laser de longuenr d'onde $1,05 \mu \mathrm{m}$, de durée 1,3 ns et d'intensité $5,44 \quad 10^{11}$ W/cm2.

Les valeurs maximales de ces contraintes sont mesurées pour différentes épaisseurs d'échantillon et sont données en fonction de l'éclairement laser et de deux dunées d"irradiation sur les figures 2 et 3 pour l'AU4G et figures 4 et 5 pour le cuivre.

contrainte (kbars)

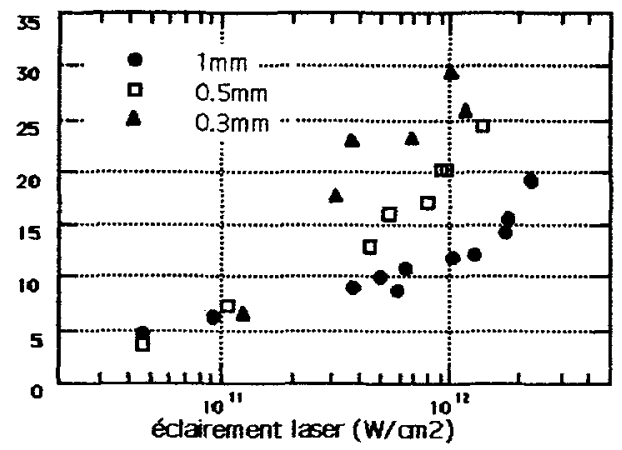

Figure 2 : Contraintes maximales dans la jange à quartz pour une cible dAU4G à $t=1.3$ ns contrainte (kbars)

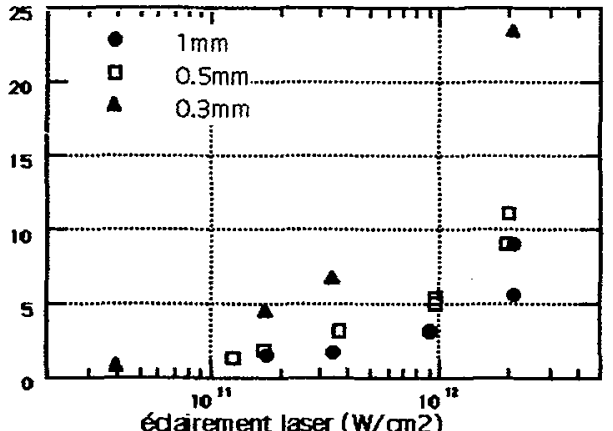

Figure 4 : Contraintes maximales dans la jange à qrartz pour une cible de cuivre à $t=1.3$ ns

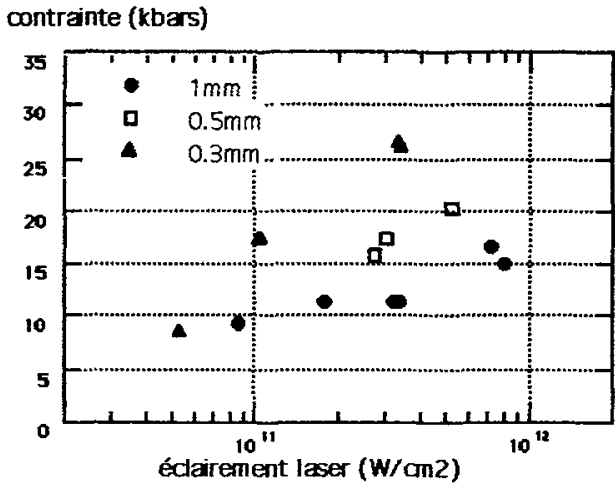

Figure 3 : Contraintes maximales dans la jauge à quartz pour une cible d'AU4G à $t=4$ ns contrainte (kbars)

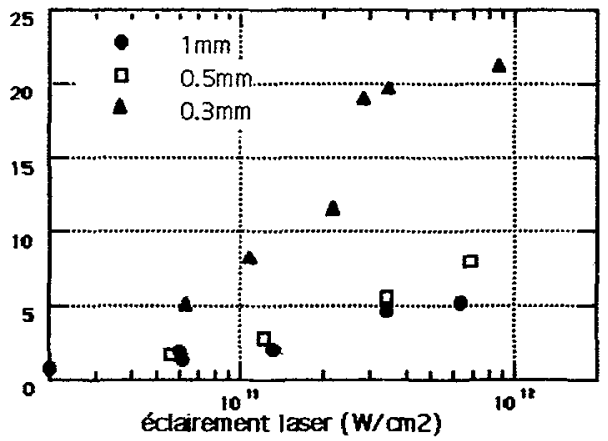

Figure 5 : Contraintes maximales dans la jauge à quartz pour une cible de cuivre à $t=4$ ns 
Deux exemples de cibles endommagées aprés irradiation sont illustrées sur les photos suivantes.
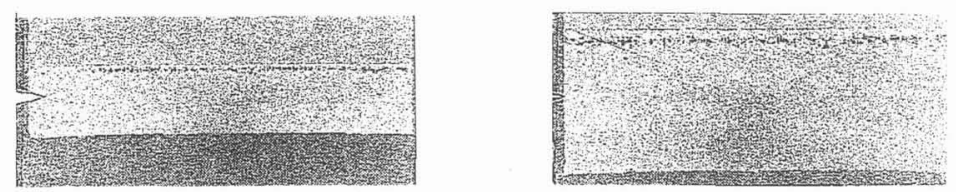

$$
\mathrm{G}=18
$$

Cuisre pur de type O. F. H. C. $(\mathrm{Cu} \geq 99,95 \%) \mathrm{c}=\mathrm{Imm}$

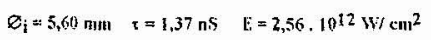

$$
G=36
$$

Bulles d'endommagenrent a -85 $4 \mathrm{~m}$ de la surface.
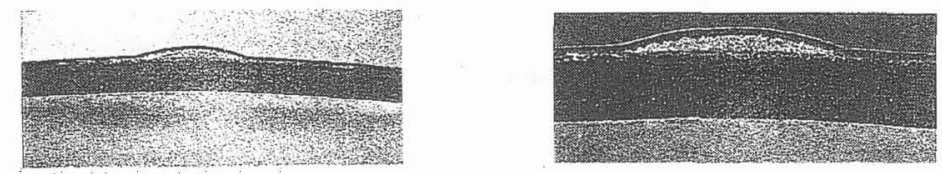

$G=18$

Cuivre pur de type O. F. H. C. ( $\mathrm{C} u \geq 99,95 \%) \quad e=0,5 \mathrm{mur}$

$\varnothing_{\mathrm{i}}=5,60 \mathrm{~mm} \quad \tau=4,42 \mathrm{nS} \quad E=9,66.10^{11} \mathrm{~W} / \mathrm{cm}^{2}$

$G=36$

Epaisseur d'écaille $-65 \mu \mathrm{m}$

Les états d'endommagement en fonction de l'éclairement laser sont résumés pour l'aluminium sur la figure 6 et pour le cuivre sur la figure 7 .
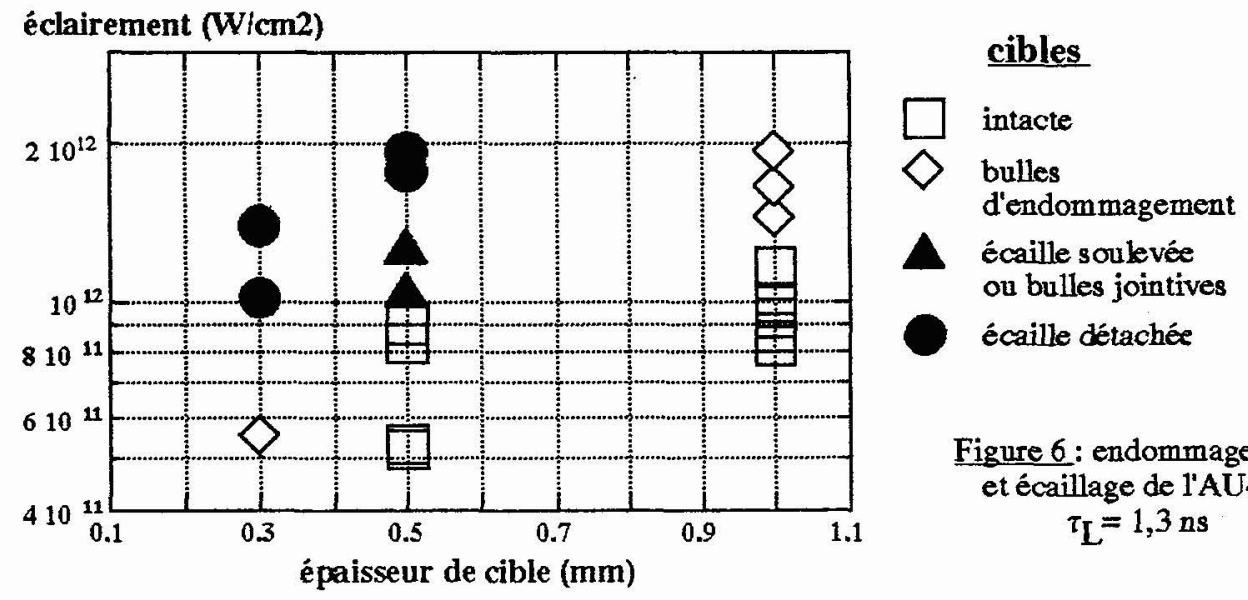

Figure 6: endommagement et écaillage de l'AU4G.

$$
\tau_{\mathrm{L}}=1,3 \mathrm{~ns}
$$

\section{cibles}

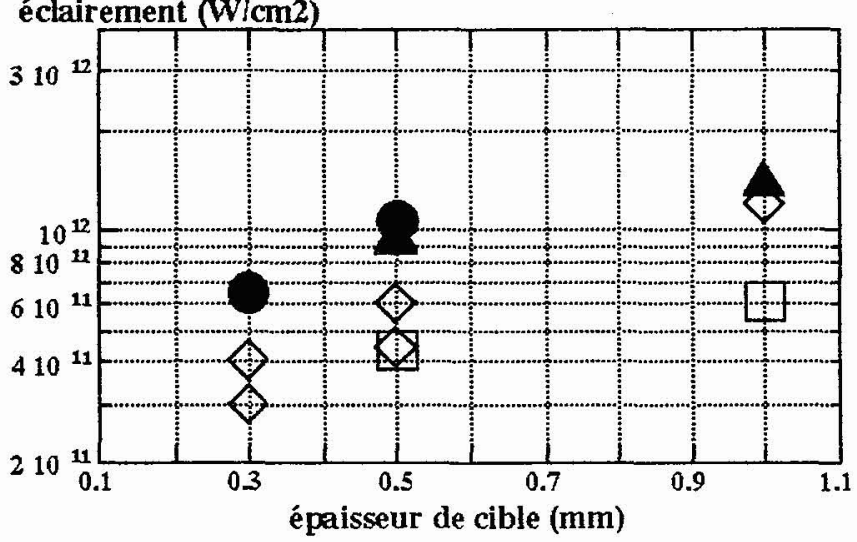

intacte

bulles

d'endommagement

écaille soulevée ou bulles jointives

écaille détachée

Figure 7 : endommagement et écaillage du cuivre.

$$
\tau_{\mathrm{L}}=4 \mathrm{~ns}
$$




\section{Simulations numériques des expéríences:}

L'exploitation des résultats expérimentaux précédents a été effectuée à l'aide des codes monodimensionnels FCI1 et EFFET1 qui traitent:

- le dépôt d'énergie laser dans les phases solide, liquide, vapeur et plasma et la formation du choc (3) : l'absorption du rayonnement laser en phase solide et liquide est fortement dépendante de la réflexion de surface. Nous employ ons le modèle de Drude-Zener ainsi que la contribution interbande pour évaluer le coefficient de réflexion en fonction de la température. En phases vapeur et plasma, l'absorption laser se fait par bremsstrahlung inverse électron-neutre et électron-ion, le degrê d'ionisation du milieu étant décrit à partir des tables SESAME de Los Alamos;

- la propagation et l'atténuation du choc résultant de ce dépôt: à l'aide d'équations d'état multiphases et d'un modèle élasto-plastique parfait ou d'un modèle S.C.G avec le critère de Von Misès ;

- l'endommagement et l'écaillage suivant différents modèles:

- tension limite

- Tuler-Butcher (modèle cumulatif) (4)

- N.A.G de Curran, Seaman, Shockey (5)

Ils permettent ainsi de traiter en une seule séquence les différents phénomènes physiques depuis le dépôt d'énergie laser jusqu'à l'endommagement.

La figure 8 représente la formation du thermochoc dans la zone du dépôt d'ênergie laser pour une cible d'ahuminium. La propagation et l'atténuation de cette contrainte dans l'épaisseur du matériau est représentée sur la figure 9. La forte atténuation constatée est dûe à la brièveté de l'impulsión laser. Les épaisseurs de matériau sont suffisantes pour mettre en évidence le détachement du précurseur élastique. On remarque aprés réflexion de la contrainte sur la face arrière de l'échantillon, la propagation d'une tension qui peut provoquer l'écaillage.

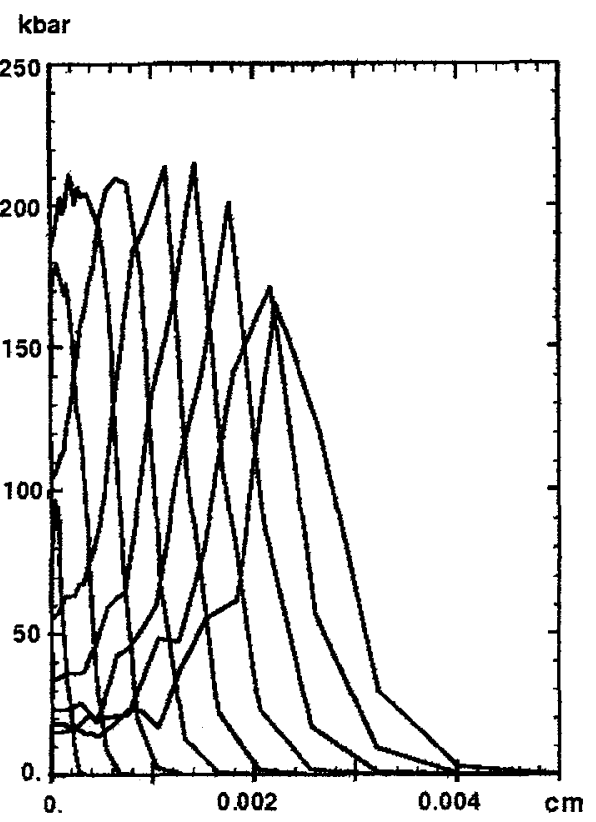

Fig 8 : Naissance du thermochoc: volution de la contrainte entre 0 at 5 ns toutes les $0,5 \mathrm{~ns}$

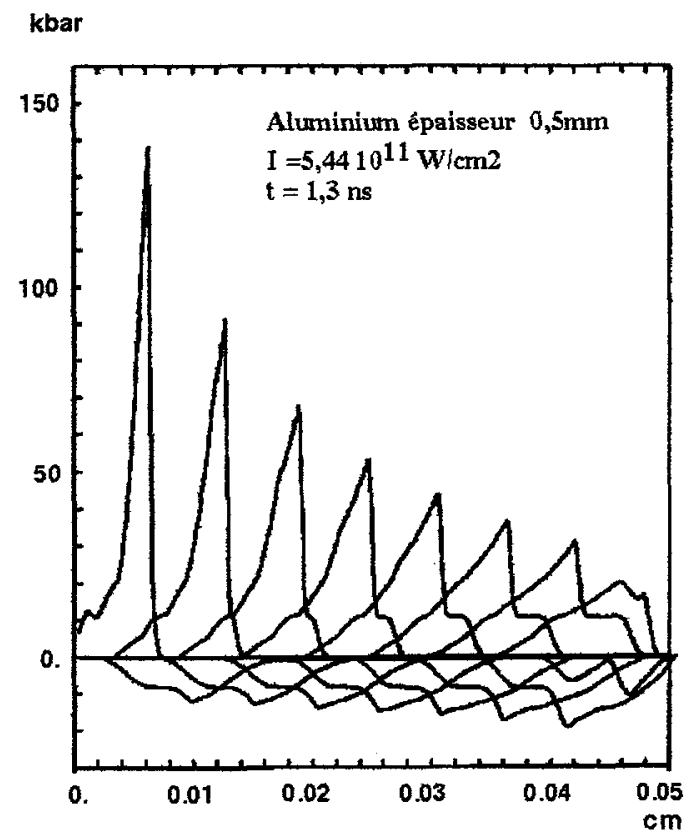

Fig 9 : propagation du choc dans l'épaisseur dn matériat: évolution de ha contrainte tontes les 10 ns

La figure 10 présente la comparaison entre la mesure et le calcul de la contrainte dans la jauge à quartz. On constate une bonne concordance, tant d'un point de vue chronométrie que de la forme et de l'amplitude du signal. 


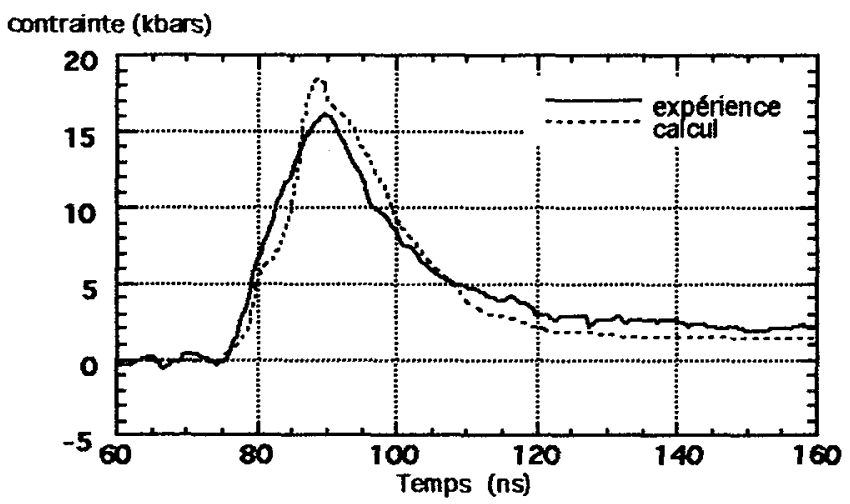
Figure 10:
Comparaison experience-calcul.
Evolution temporelle de ba
contrainte à l'arrière d'une cible
d'aluminium de 0,5 mm
d'́́paisseur irradíe par une
impulsion laser de longueur
d'onde $1,05 \mu \mathrm{m}$, de duré 1,3 ns
et d'intensite $5,4410^{11} \mathrm{~W} / \mathrm{cm} 2$.

Les expériences avec écaillage dans l'aluminium ont été exploitées dans le but de vérifier si les paramètres généralement utilisés dans les modèles d'écaillage rendaient compte numériquement des résnltats expérimentaux, en particulier des épaisseurs écailkées. Deux modèles d'écaillage ont été testés:

- le modèle en tension limite pour lequel nous avons adopté comme valeur de la tension d'écaillage $\sigma_{\text {frac }}=25 \mathrm{kbars}(6)$;

- le modèle cumulatif de Tuler-Butcher $\int_{\text {tonil }}^{1}\left(\sigma-\sigma_{\text {seril }}\right)^{\lambda} \mathrm{dt}=K$ avec les paramètres suivants : $\sigma_{\text {seuil }}=4,25$ kbars $; \lambda=2,02 ; K=3,9810^{13}$ cgs obtenus pour des conditions expérimentales et des matériaux similaires aux nôtres (7) (8).

Les calculs sont effectués en faisant varier les différents paramètres mis en jeu dans l'expérience : éclairement laser, durée de l'impulsion, épaisseur de la cible.

\section{- Modele en tension limite :}

Les épaisseurs écaillées en fonction du flux, obtenues par le calcul sont comparées aux résultats expérimentaux sur la figure 11 pour une épaisseur d'aluminium de $0,3 \mathrm{~mm}$ et une durée d'irradiation de 1,3 ns. Les épaisseurs d'écaille calculées varient peu avec l'éclairement laser et rendent compte des valeurs expérimentales : la contrainte d'écaillage de l'AU4G $\sigma_{\text {frac }}=25$ kikbars est satisfaisante.

\section{- Modele de Tuler-Butcher:}

Les résultats des calculs comparés à l'expérience sont présentés sur la figure 12 pour une intensité laser de $210^{12} \mathrm{~W} / \mathrm{cm}^{2}$ et une durée d'irradiation de $1,3 \mathrm{~ns}$. On constate que les valeurs expérimentales et calculées sont voisines. Pour des épaisseurs de cible supérieures à $0,8 \mathrm{~mm}$, les résultats du calcul indiquent qu'il n'y a pas de fracture, alors que l'expérience montre une ligne d'endommagement préfigurant la fracture. Cette différence peut être attribuée à la rusticité du modèle théorique qui ne prend pas en compte l'endommagement. On peut cependant conclure que les paramètres utilisés dans le modèle de Tuler-Butcher permettent de restituer correctement les épaisseurs d'écaille obtenues dans l'expérience.

Epaisseur écaille (micron)

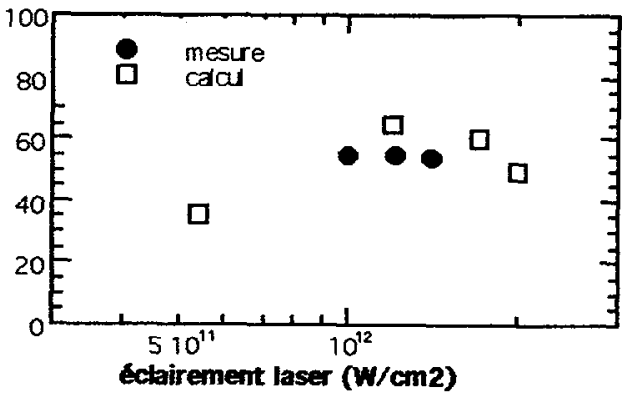

Figare 11: Epaisseur d'6caillo en fonction de l'6chairement laser - Comparaison expériences - calculs - Aluminium d'Epaissear $0,3 \mathrm{~mm}$ - Duré d'irradiation $t=1,3 \mathrm{~ns}$ - Longueur d'onde $\lambda=1.05 \mu \mathrm{m}$ épaisseur écaille (micron)

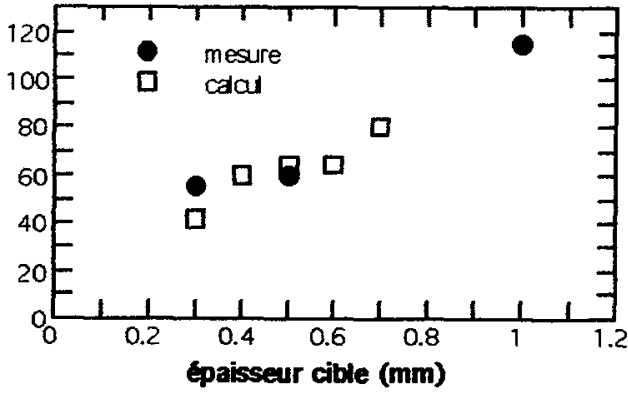

Figure 12: épaisseur d'ecaille on fonction de l'epaissear de h cible d'aluminium - Comparaison expériences - calculs.. Intensité laser $I=210^{12} \mathrm{~W} / \mathrm{cm}^{2}$ - Dunbe dirradiation $t=1,3 \mathrm{~ns}$ Longueur d'onde $\lambda=1,05 \mu \mathrm{m}$ 


\section{Conclusion:}

Nous avons présenté un grand nombre de résultats expérimentaux sur les contraintes et les écaillages induits par un dépôt d'énergie laser dans des cibles d'aluminium et de cuivre.

Une première interprétation numérique de ces résultats a permis de restituer de façon satisfaisante les contraintes sur la face arrière de l'échantillon mesurées par une jauge à quartz. On peut en déduire que les modèles utilisés pour le calcul du dépôt laser, pour la formation, la propagation et l'atténuation des contraintes sont représentatifs de la réalité.

D'autre part, nous avons montré que les paramètres généralement utilisés dans les modèles d'écaillage (en tension limite et Tuler-Butcher) restituaient correctement les épaisseurs d'écailles obtenues expérimentalement dans l'aluminium.

La poursuite de cette étude consiste à exploiter les résultats expérimentaux obtenus pour d'autres conditions expérimentales avec l'aluminium et à interpréter numériquement les résultats sur le cuivre.

\section{Remerciements :}

Nous tenons à remercier : Mesdames C. Reisse et $\mathrm{F}$. Wagon pour les développements numériques du code FCI1, Monsieur J.L. Loubat pour la réalisation des montages expérimentaux et l'analyse des cibles aprés tir, Monsieur J.C. Wettling pour la mise au point de diagnostics et sa participation aux dépouillements ainsi que les équipes de conduite des expériences pour la mise en ceuvre de l'ensemble de ces essais.

\section{Références:}

(1) J. David, J.C. Wettling, P. Combis, G. Niérat, M. Rostaing -" Quartz Gauge and ballistic penduhum measurements of the mechanical impulse imparted to a target by a laser pulse "-8th International Symposium on Gas Flow and chemical Lasers.Madrid 10-14 september 1990.SPIE Vol.1397. p 697(1991)

(2) P. Combis, B. Cazalis, J.David, A. Froger, M. Louis-Jacquet, B. Meyer, G. Niérat, A. Salères, G.Sibille, G. Thiell and F. Wagon - " Low Fluence laser target coupling "- Laser and Particle Beams (1991) vol $9 n^{\circ} 2$ p 403-420.

(3) A. Salères, B .Cazalis, P. Combis, J. David, B. Meyer, G. Niérat, G. Sibille,G.Thiell et F. Wagon"Couplage thermique et mécanique dans l'interaction laser-matière à éclairement modéré ". Revue Scientifique et technique de la Défense- 4ème trimestre 1992 p 77-99.

(4) B.M. Butcher, L.M. Barker, D.E. Munson, C.D. Lundergan - "Influence of Stress History on TimeDependent Spall in Metals". A.I.A.A J., 2, 977 (1964)

(5) L. Seaman, T.W. Barbee, and D.R. Curran, "Dynamic Fracture Criteria of Homogeneous Materials", AD 893701, Stanford Research Institute, Menlo Park, California, December 1971.

(6) V.E. Fortov, V.V. Kostin, S. Eliezer. - " Spallation of metals under laser irradiation ". J. A. P 70 (8), 15 October $1991 \mathrm{p} 4524$.

(7) F.R. Tuler, B.M. Butcher- "A Criterion for the time dependence of Dynamic Fracture". Inter. Journ. of Fracture Mechanics, 43 (16)

(8) M. Boustie, F. Cottet. " Experimental and numerical study of laser induced spallation into Aluminum and Copper targets " - JA.P 69 (11), 1 June 1991, p 7533. 